\title{
Long term outcomes of one-stage augmentation anterior urethroplasty: a systematic review and meta-analysis
}

\author{
Cooper R. Benson ${ }^{1}$, Gen Li ${ }^{2}$, Steven B. Brandes ${ }^{1}$ \\ ${ }^{1}$ Department of Urology, Columbia University Medical Center, New York, NY, USA; ${ }^{2}$ Department of \\ Biostatistics, Columbia University Medical Center, New York, NY, USA
}

\section{ABSTRACT}

Objective: The objective is to summarize and characterize the long-term success of anterior augmentation urethroplasty (AU) in published series. The current literature on AU consists largely of retrospective series reporting intermediate follow-up and incompletely characterize the long term outcomes of AU.

Materials and Methods: A systematic literature review was performed consistent with PRISMA guidelines to characterize long-term outcomes of AU with a minimum upper limit follow-up of 100 months. Penile/preputial skin flaps and graft and oral mucosal graft urethroplasties were included. The primary outcome was stricture-free survival for one-stage AU. Secondary analysis evaluated differences in outcomes based on two failure definitions: the need for intervention versus presence of recurrent stricture on cystoscopy or urethrography. Hazard rates were induced from the reported failure rates of one-stage AU and fixed and random effect models were fitted to the data. Additional subset analysis, removing potential confounders (lichen sclerosus, hypospadias and penile skin graft), was performed.

Results: Ten studies met inclusion criteria, and two studies reported separate outcomes for grafts and flaps, and thus were included separately in the analysis. The mean hazard rate across all studies was 0.0044 , the corresponding survival rates at 1 year $0.948,5$ years $0.766,10$ years 0.587 , and 15 years 0.45 . Subset analysis of the 4 select and homogeneous studies noted 1, 5, 10, and 15 years survival rates of 0.97, 0.96, 0.74, and 0.63 , respectively.

Conclusions: The long-term success rates of augmentation urethroplasty are appear to be worse than previously appreciated and patients should be counseled accordingly.

\section{ARTICLE INFO}

Cooper R. Benson

http://orcid.org/0000-0001-7572-954X

Keywords:

Urethral Stricture; Systematic

Review [Publication Type];

Cystoscopy

Int Braz J Urol. 2020; 47: 237-50

Submitted for publication:

March 27, 2020

Accepted after revision:

April 01, 2020

Published as Ahead of Print:

April 20, 2020

\section{INTRODUCTION}

Urethroplasty is the gold standard treatment for urethral strictures, which is cost effective and has the highest reported success rates (1). There are a large variety of urethroplasty techniques that are utilized in modern practice. Nevertheless, regardless of the technique, the shared goals are of safety (minimal side effects), efficacy (unobstructed urethra with normal voiding), and durability (long-term effective results). Urethral reconstructive surgery decision making is highly nuanced and requires consideration of a multitude of factors, including stricture characteristics (length, location, 
and etiology), prior treatments, surgeon experience, co-morbidities and availability/quality of penile skin or graft material (2). Clearly, the surgical techniques and choice of graft/flap materials for augmentation urethroplasty (AU) have evolved over-time, and there is not a one-size fits all approach. Excision and primary anastomosis (EPA) urethroplasty has the highest reported durable success rates; (>85\%); however, this is not always possible and often have to rely on buccal mucosal grafts (BMG) and/or penile skin flaps for successful reconstruction (3).

For AU, patients are often quoted an average success rate approximating 85\% for augmentation urethroplasty. This is based on intermediate follow-up ( 5 years), with variable reported definitions of success (2). Moreover, the ICUD consensus statement on urethral strictures, prominently states that average success rates for one-stage bulbar urethroplasty is $83-88.8 \%$, Asopa $86.7 \%$, one-stage penile 75\% and panurethral $88.2 \%(2,4)$. While the durability of EPA urethroplasty is well documented, the long term success of AU has been poorly characterized. Thus, raising the question, is an $85 \%$ success rate an accurate representation of long-term AU success? We sought to characterize and analyze and summarize the long-term outcomes (10 and 15 year) of AU in literature. Our hypothesis is that the long-term outcomes of AU are well below the commonly quoted 85\%.

\section{MATERIALS AND METHODS}

\section{Search Strategy and Study Selection}

A systemic literature review using Pubmed/MEDLINE and Embase databases from 20002018 was performed consistent with the Preferred Reporting Items for Systematic Reviews and Meta-Analyses (PRISMA) guidelines, in order to identify long-term outcomes and prognosis of augmentation urethroplasty (5). Terminology utilized for the database search included: Urethroplasty and long term outcomes, long term follow-up and urethroplasty, long term and urethroplasty, urethroplasty and outcomes, substitution urethroplasty, AU and outcomes, outcomes and urethral stricture. Inclusion criteria for meta-analysis included all studies reported in the adult literature on one-stage AU for anterior urethral strictures. Further inclusion criteria were studies that included patients who had follow-up of at least 100 months, and urethral reconstruction utilizing BMG, preputial/penile skin grafts and/or flaps. We included studies utilizing Palminteri urethroplasty, as well as Asopa inlay urethroplasty techniques $(6,7)$. The term "augmentation" urethroplasty was selected to encompass all of these techniques in accordance to the ICUD terminology consensus statement (8). We excluded studies and patients who underwent augmented anastomotic urethroplasty or staged urethroplasties, had the upper limit range of follow-up $<100$ months, and/or non-BMG and non-penile skin graft/ flap urethroplasties. Manuscripts without English translation were also excluded from the analysis as well as papers with incomplete or non-granular data.

Figure-1 demonstrates the PRISMA flow diagram and how studies were selected. We identified 1.302 unique articles from our search, of which 103 abstracts were reviewed, the remainder of the studies were excluded based largely on titles of the studies that were not relevant to the meta-analysis. Of the 103 abstracts reviewed in detail, only 34 abstracts included patients undergoing AU with adequate follow-up or unclear follow-up, based on abstract alone and these 34 manuscripts were reviewed by two authors (CRB, SBB). We identified 10 retrospective studies from expert urethral surgeon series, that met inclusion criteria, and classified as level 3 evidence.

\section{Data Extraction}

When present, we extracted the following variables: number of patients, types of urethroplasty, substitution material, length of follow-up, definitions of success, Kaplan-Meier method censor points for stricture-free survival, failure rates, graft/flap location. Unpublished granular data was obtained from the author of one study (9) and was included in the analysis. Additionally, two studies reported outcomes of grafts and flaps separately, and thus were individually analyzed $(9,10)$. Several studies reported outcomes on non-AU one-stage reconstruction and these patients were excluded from analysis. If this was not possible because the lack of granularity, the entire study was excluded from analysis.

\section{Outcome Measures}

The primary outcome was prognostic stricture free survival (SFS) of one-stage AU. All included 
Figure 1 - PRIMSA Diagram for inclusion and exclusion of studies in the meta-analysis.
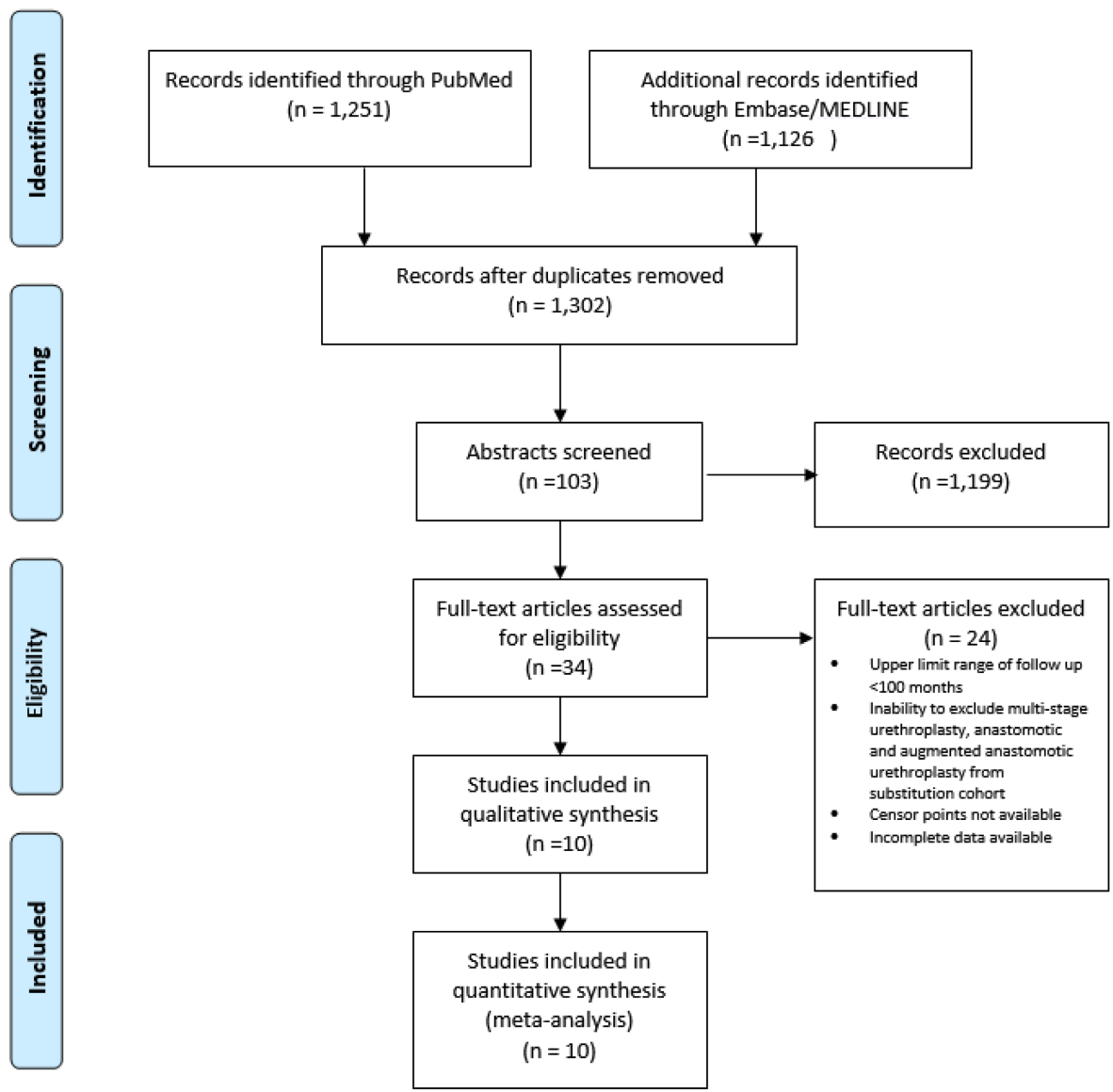

studies did not utilize the same failure definition; 6 studies utilized the need for intervention following urethroplasty and 4 studies used presence of recurrent stricture on cystoscopy or urethrography during routine follow-up. Secondary analysis compared differences in SFS based on these definitions. An additional subset analysis was performed to eliminate potential confounders known to impact urethroplasty success, in an effort to create a "best case scenario." In order to reduce contamination, we excluded patients and/or studies, when the data was not granular, that included patients with hypospadias and/or lichen sclerosus stricture etiologies, as well as the use of penile/preputial skin grafts.

\section{Statistical analysis}

The software package "metaphor" (Version 1.9-9) in R (Version 3.2.1) was used to conduct the meta-analysis on hazard rates. Hazard rates were induced from the reported failure rates of one-stage substitution anterior urethroplasty assuming survival followed an exponential distribution, and also using the above assumptions allowing us to combine the data. Random effects models were fitted to the data. Forrest plots were included to show effect sizes and confidence intervals for individual studies and also for the meta-analysis, as well as comparing the two definitions of failure. I2 analysis was used to represent heterogeneity among the studies and funnel plots were reported to represent the likelihood of publication bias in each analysis.

\section{RESULTS}

Supplementary Table- 1 summarizes the granular data from these 10 studies $(3,6,9-16)$. Across the studies, a total of 954 patients were included for analysis. Figure-2A demonstrates the hazard rates of all included studies. The average hazard 
Figure 2 - A) Forrest Plot of all studies included in analysis demonstrating the effect size of each study as hazard rates, which captures time to stricture recurrence in each individual study. B) Forrest Plot of Studies, demonstrating the effect size of each study using the need for instrumentation after urethroplasty as definition of failure. C) Forrest Plot of studies, demonstrating the effect size of each study, using recurrent stricture on routine urethrography or cystoscopy as definition of failure.

A

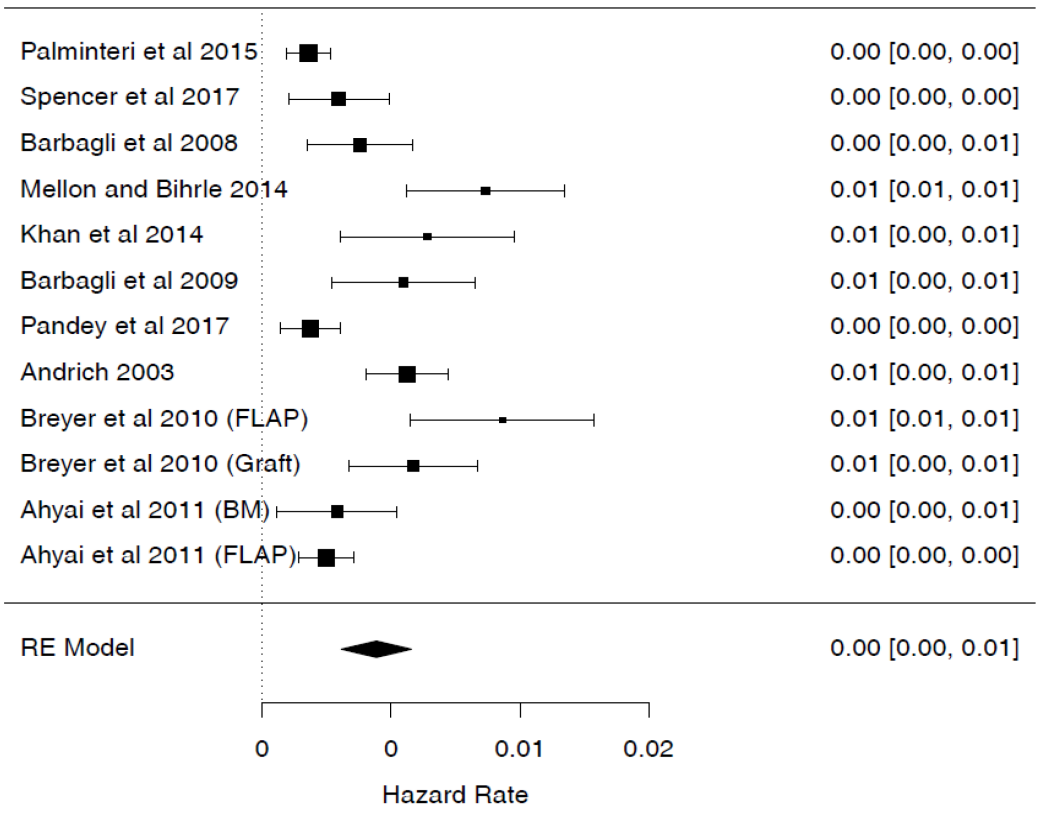

B

\begin{tabular}{|c|c|c|c|}
\hline Palminteri et al 2015 & $r$ & & $0.00[0.00,0.00]$ \\
\hline Spencer et al 2017 & $\longmapsto$ & & $0.00[0.00,0.00]$ \\
\hline Barbagli et al 2008 & $\longmapsto$ & & $0.00[0.00,0.01]$ \\
\hline Mellon and Bihrle 20 & $\longmapsto$ & & $0.01[0.01,0.01]$ \\
\hline Barbagli et al 2009 & $\longmapsto$ & & $0.01[0.00,0.01]$ \\
\hline Breyer et al 2010 (F) & $A P)$ & & $0.01[0.01,0.01]$ \\
\hline Breyer et al 2010 (G) & $\longmapsto$ & & $0.01[0.00,0.01]$ \\
\hline \multirow[t]{2}{*}{ RE Model } & & & $0.01[0.00,0.01]$ \\
\hline & $\begin{array}{cc}0 & 0.01 \\
\text { Hazard Rate }\end{array}$ & 0.02 & \\
\hline
\end{tabular}

C

\begin{tabular}{|c|c|c|}
\hline Khan et al 2014 & $\longrightarrow$ & $0.01[0.00,0.01]$ \\
\hline Pandey et al 2017 & $\longmapsto$ & $0.00[0.00,0.00]$ \\
\hline Andrich 2003 & $\longmapsto$ & $0.01[0.00,0.01]$ \\
\hline Ahyai et al 2011 (BM) & $\longmapsto$ & $0.00[0.00,0.01]$ \\
\hline Ahyai et al 2011 (FLAP) & $\longmapsto$ & $0.00[0.00,0.00]$ \\
\hline \multirow[t]{2}{*}{ RE Model } & 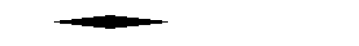 & $0.00[0.00,0.01]$ \\
\hline & $\begin{array}{lll}0 & 0 & 0.01 \\
\text { Hazard Rate } & & 0.01\end{array}$ & \\
\hline
\end{tabular}


rate across all studies was 0.0044 (SD 0.000693), which corresponds to a 5-year survival rate of 0.766 (95\% CI 0.706-0.831). Further, the survival rates at 1 year were 0.948 (95\% CI 0.933-0.964), at 10 years, 0.587 (95\% CI $0.499-0.691)$, and at 15 years, 0.45 (95\% CI 0.352-0.574), as shown in the Kaplan-Meier curve in Figure-3. Two studies, Breyer et al. and Ahyai et al., reported separate outcomes for each fasciocutaneous skin flap and BMG patients, and thus were included separately in our analysis $(9,10)$.

The outcomes of urethroplasty were compared based on the definition of urethroplasty failure and found that the outcomes were not significantly different using either definition $(p=0.132)$ (Figures 2B and 2C). We identified significant heterogeneity among the included studies in the meta-analysis ( $\mathrm{I} 2=85.66 \%, \mathrm{p}<0.001)$. Additionally, we identified publication bias among the included studies all together and when divided by failure definition, which is demonstrated in the funnel plots in supplementary Figure-1.

The subset analysis for the "best case scenario" included only four studies that met the criteria. Qualitative synthesis of the data from these four studies demonstrated corresponding survival rates 0.969 (95\% CI 0.956-0.983) at 1 year, 0.857 (95\% CI 0.798-0.920) at 5 years, 0.735 (95\% CI $0.638-0.847)$ at 10 years, and $0.630(95 \% \mathrm{CI}$ $0.509-0.779$ ) at 15 years (Figure-4A) and the corresponding funnel plot is shown in Figure-4B. There was a significant difference in SFS at all time points, when comparing these 4 "best case scenario" studies to the remaining 6 studies $(\mathrm{p}=0.0011)$.

\section{DISCUSSION}

To our knowledge, this is the first study to combine expert series as a meta-analysis to evaluate the long-term success of AU. The current literature

Figure 3 - Estimated Kaplan-Meier curve overlay on the survival rates from each study included in the meta-analysis.

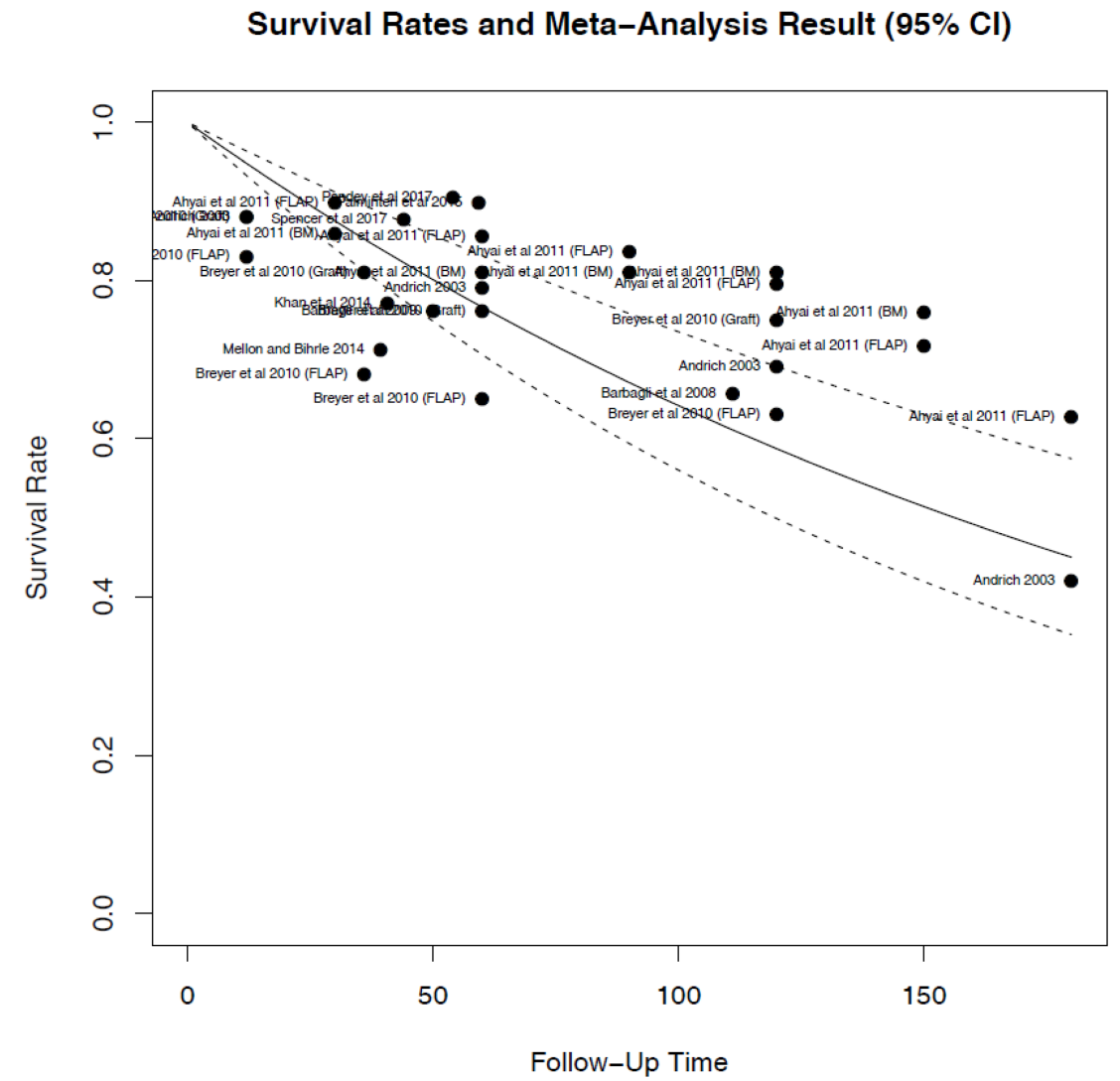


Figure 4 - A) Sub-Analysis- Estimated Kaplan-Meier curve overlay on the survival rates from each study included in the meta-analysis, when excluding studies using penile skin grafts and reporting patients with a history hypospadias and lichen sclerosus. B) Funnel Plots of the same studies, indicating less publication bias than seen in the full analysis.

A Survival Rates and Meta-Analysis Result $(95 \% \mathrm{Cl})$
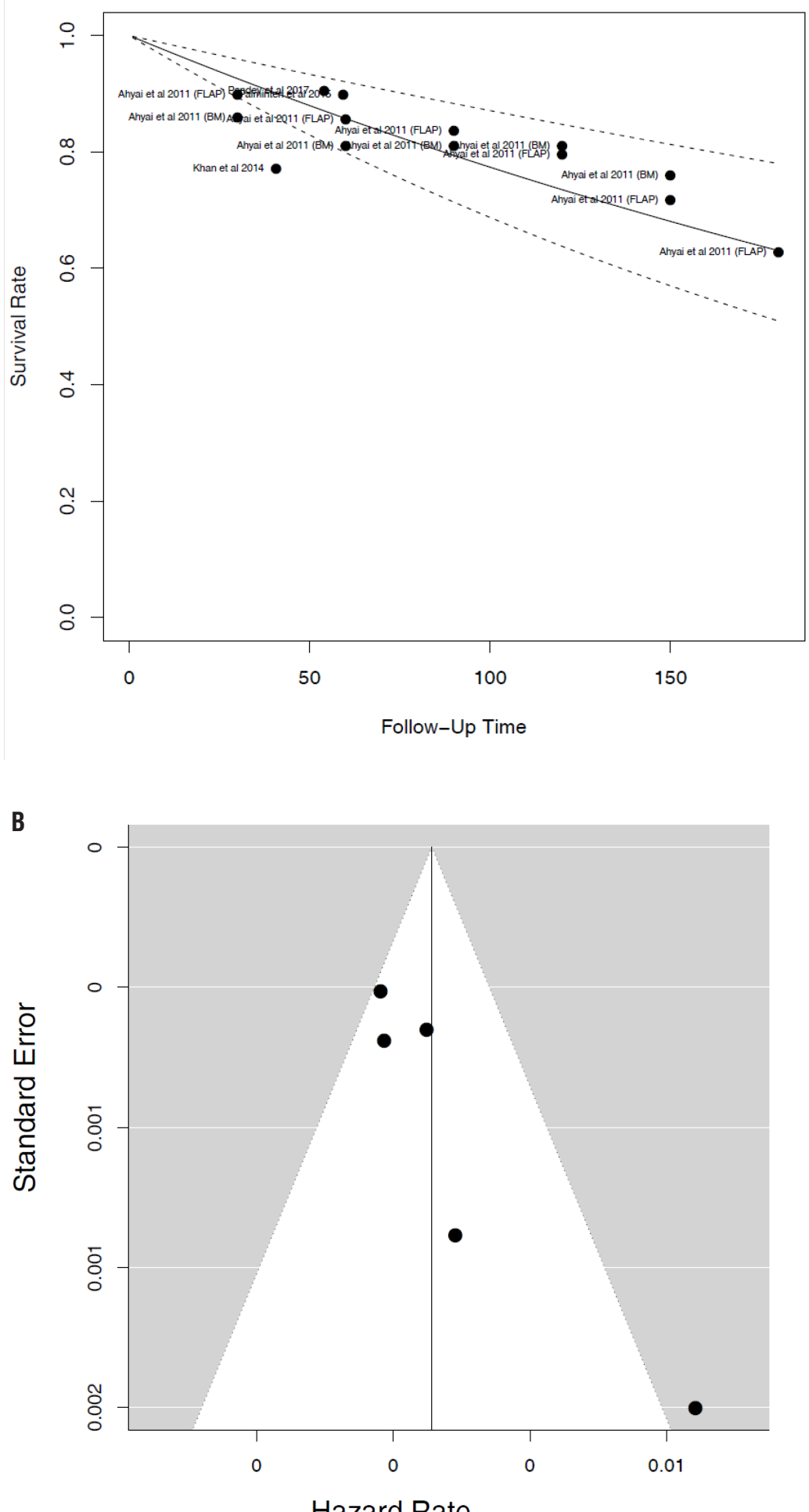

Hazard Rate 
is deficient in reporting the long-term outcomes beyond five years for AU, largely low level of evidence from high volume expert surgeons and centers $(2,4)$. Certainly, it is well understood that at intermediate follow-up (5 years), urethroplasty success far exceeds that of endoscopic treatments. However, this data questions the long-term durability of these results for AU and suggests worse SFS than publish with intermediate follow-up.

In order to contextualize our results and meta-analyze the data, we relied on published outcomes of urethroplasty techniques that enabled us to combine the data for analysis. Vasudeva et al. randomized 80 patients to have either dorsal or ventral BMG onlay urethroplasty with non-inferior success rates, 92.5\% versus 90\% respectively (17). Similarly, Aldagadossi et al. randomized patients to dorsal onlay or inlay BMG urethroplasty and found no difference in success rates, $88 \%$ versus $86.4 \%$ (18). Furthermore, a recent systematic review also noted comparable success rates of $88 \%$ at 3 years, regardless of graft location (4). Thus, indicating that outcomes, regardless of BMG placement locations, are non-inferior. Additionally, there have been two randomized trials comparting dorsal BMG and penile skin flaps, demonstrating non-inferior success rates. Dubey et al. published success rates of $89.9 \%$ versus $85.6 \%$, respectively, and similarly Soliman et al. found similar results with success rates of $89.5 \%$ versus $83.3 \%$ for BMG compared to penile skin flaps, respectively $(19,20)$. Lastly, there are several series demonstrating similar outcomes of free penile/preputial skin grafts AU compared to BMG AU. Alsikafi et al. reported similar success for penile skin graft (84\%) and BMG AU (87\%) $(\mathrm{p}=0.7375)(21)$. This was also corroborated by Raber et al., which found no significant difference in bulbar urethroplasty success comparing BMG and penile skin grafts (22). This is further supported by a systematic review, revealing recurrence rates of $14.5 \%$ and $15.7 \%$ for grafts and flaps respectively (23). In contrast, a recent systematic review evaluated urethroplasty outcomes related to BMG versus penile skin grafts and found higher interim success of BMG (85.9\%) compared to penile skin grafts $(81.8 \%)(\mathrm{p}=0.01)$ (24). However, our analysis was not powered to differentiate outcomes between penile skin/preputial flaps, penile skin/preputial grafts and BMG in urethral reconstruction. While objective and anatomic outcome measures are important, it is also essential to evaluate the quality of life and subject outcomes of urethral reconstruction.

Symptomatic urethral strictures interfere with voiding function and can have a profound impact on patients' quality of life and the patient's family. The published definitions of success vary widely and include uroflowmetry, narrowing on cystourethroscopy or urethrography, calibration with bougienage, need for endoscopic intervention, as well as patient reported outcome measures (PROM) $(1,25)$. Bertrand et al. found that patient satisfaction based on PROM was strongly associated with objective measures of urethroplasty success (26). Moreover, there is a recent impetus to include PROMs as a measure of urethroplasty success, furthering the emphasis on patient satisfaction (27). The most common practice pattern among GURS members is to define urethroplasty failure as the "need for secondary procedures" and the most common methods for screening for stricture recurrence are uroflowmetry and post-void residual (25). However, invasive testing, such as routine cystoscopy (19\%) and urethrography (17\%), are less commonly utilized as routine screening methods by GURS members (25). Urethral surgery is mostly a quality of life surgery, and thus is successful if the patient reports improved quality of life and voiding function. This supports the notion that invasive testing is often overly aggressive and will not alter management, in the asymptomatic and satisfied patient.

Moreover, Baradaran et al. reported no difference in validated questionnaire scores between patients with normal cystoscopy (anatomic success), $<17 \mathrm{~F}$ and $>17 \mathrm{~F}$ recurrence of stricture (27). Among those with $<17 \mathrm{~F}$ recurrent stricture, 66\% of the patients did not require secondary interventions, implying there was still subjective success and improved quality of life despite cystoscopic findings (27). Furthermore, our analysis demonstrated no significant difference in long-term outcomes comparing the definitions of success $(p=0.132)$. This supports defining urethroplasty 
success, as the need for secondary intervention; however, the meta-analysis was not powered to answer such question.

As reconstructive urologists, patients are often quoted success rates of around 85\% (75.6\%90.6\%) for AU, which may be inflating its durability $(2,4)$. Furthermore, urethroplasty surgery is associated with a significant learning curve, between 100 and 400 cases, before achieving consistent successful outcomes $(28,29)$. Inexperienced urethral surgeons will likely not have the same results as the results in expert published series. A recent population analysis of urethroplasty outcomes, demonstrated a 32\% failure rate for anterior urethroplasty at 3 years and trending toward $40 \%$ failure rate at 5 years, which highlights that community performed urethroplasty outcomes are worse than the $85 \%$ interim results by expert surgeons (30). The results of expert surgeon series are not necessarily reproducible by the occasional urethral surgeon. Thus, acknowledging this information is an essential aspect of the preoperative urethroplasty informed consent process. This analysis suggests a progressive deterioration rate over time for AU, which has been observed in multiple urethroplasty series $(9,10,14,31,32)$. Several series have indicated that most failures occur within the first two years following urethroplasty; however, not all urethroplasty failures are equivalent $(10,14,31)$. There are several potential explanations for AU failure; under-estimation of stricture length or degree of spongiofibrosis, failure of the graft to take due to poor host bed conditions, or flap loss due to compromised pedicle. This analysis is limited by lack of detail of failure patterns for AU, and thus are not able to incorporate this into our analysis.

We feel our conclusions about long-term success of AU are reliable even with the inherent limitations. After removing potential confounders in our subset analysis, the 15-year stricture free survival went from 45\% to 63\% for AU, which still falls short of the quoted 85\% success at intermediate follow-up. Future studies would attempt to prospectively evaluate patients with similar stricture characteristics, risk factors, and surgeon experience and follow these patients' long term. Furthermore, it is critical to better understand and identify the causes of progressive attrition in AU with long-term follow-up. Several potential causes of late failure include: an under-appreciation of the degree of spongiofibrosis at the time of reconstruction, progressive spongiofibrosis over time, and/ or subsequent urethral trauma leading to recurrence. Perhaps advanced imaging technology such as MRI or ultrasound elastography, will be enable more accurate characterization of spongiofibrosis that may not be readily apparent preoperatively or intraoperatively.

Our study limitations are merely a reflection of the lack of granularity of the reported outcomes of urethroplasty. The studies included herein are largely lower level of evidence and retrospective series with single surgeon or multiple expert surgeon series, and thus largely just observational and very heterogenous. Furthermore, as demonstrated in the funnel plots in supplemental Figure-1, there is publication bias which may have also impacted our results. Certainly, bias is also introduced with inclusion of a heterogeneous patient population with non-uniform stricture characteristics with variability in the AU grafts and/or flaps utilized and variable definitions of success.

\section{CONCLUSIONS}

The long-term success of AU seems not as durable as reported with intermediate follow-up and appears to have continued deterioration with more than 100 months of follow-up. AU has worse than appreciated long term outcomes and further prospective studies are necessary to corroborate these findings. Our meta-analysis gives the urethral surgeon more information regarding the long-term success rates to aid in preoperative patient counseling. We cannot impart the long-term success of AU, unless we first acknowledge that long term results potentially are not as robust as once believed.

\section{CONFLICT OF INTEREST}

None declared. 


\section{REFERENCES}

1. Meeks JJ, Erickson BA, Granieri MA, Gonzalez CM. Stricture recurrence after urethroplasty: a systematic review. J Urol. 2009;182:1266-70.

2. Chapple C, Andrich D, Atala A, Barbagli G, Cavalcanti A, Kulkarni $S$, et al. SIU/ICUD Consultation on Urethral Strictures: The management of anterior urethral stricture disease using substitution urethroplasty. Urology. 2014;83(3 Suppl):S31-47.

3. Andrich DE, Dunglison N, Greenwell TJ, Mundy AR. The long-term results of urethroplasty. J Urol. 2003;170:90-2.

4. Mangera A, Patterson JM, Chapple CR. A systematic review of graft augmentation urethroplasty techniques for the treatment of anterior urethral strictures. Eur Urol. 2011;59:797-814.

5. Moher D, Shamseer L, Clarke M, Ghersi D, Liberati A, Petticrew M, et al. Preferred reporting items for systematic review and meta-analysis protocols (PRISMA-P) 2015 statement. Syst Rev. 2015;4:1.

6. Palminteri E, Lumen N, Berdondini E, Di Pierro GB, Cucchiarale G, Tenti G, et al. Two-sided dorsal plus ventral oral graft bulbar urethroplasty: Iong-term results and predictive factors. Urology. 2015;85:942-7.

7. Asopa HS, Garg M, Singhal GG, Singh L, Asopa J, Nischal A. Dorsal free graft urethroplasty for urethral stricture by ventral sagittal urethrotomy approach. Urology. 2001;58:657-9.

8. Latini JM, McAninch JW, Brandes SB, Chung JY, Rosenstein D. SIU/ICUD Consultation On Urethral Strictures: Epidemiology, etiology, anatomy, and nomenclature of urethral stenoses, strictures, and pelvic fracture urethral disruption injuries. Urology. 2014;83(3 Suppl):S1-7.

9. Ahyai, SA et al. Extended long-term results of surgery for urethral strictures. J Urol. 2011;185: e42.

10. Breyer BN, McAninch JW, Whitson JM, Eisenberg ML, Mehdizadeh JF, Myers JB, et al. Multivariate analysis of risk factors for long-term urethroplasty outcome. J Urol. 2010;183:613-7.

11. Spencer J, Blakely S, Daugherty M, Angulo JC, Martins F, Venkatesan K, et al. Clinical and Patient-reported Outcomes of 1-sided Anterior Urethroplasty for Long-segment or Panurethral Strictures. Urology. 2018;111:208-13.

12. Barbagli G, Morgia G, Lazzeri M. Dorsal onlay skin graft bulbar urethroplasty: long-term follow-up. Eur Urol. 2008:53:628-33.
13. Barbagli G.,Romano G.,De Angelis M.,Lazzeri M. 146 Comparative retrospective outcome analysis of 62 patients who underwent one-stage repair of penile urethral strictures in a single referral center experience. Eur Urol Suppl 2009; 8: 157.

14. Mellon MJ, Bihrle R. Ventral onlay buccal mucosa urethroplasty: a 10-year experience. Int J Urol. 2014;21:190-3.

15. Khan R, Kelly L, Palmer M. Substitution bulbar urethroplasty using buccal mucosal graft in dorsal onlay fashion (barbagli technique): Results for patients treated over a 10-year period in a scottish regional referral centre. Urology. 2014;84(4):S85

16. Pandey A, Borisenkov M, Barta-Kelemen A, Keller H. Redo urethroplasty after unsuccessful urethral reconstruction with buccal mucosa graft. Eur Urol Suppl. 2017; 16: e485.

17. Vasudeva $\mathrm{P}$, Nanda B, Kumar A, Kumar N, Singh H, Kumar R. Dorsal versus ventral onlay buccal mucosal graft urethroplasty for long-segment bulbar urethral stricture: A prospective randomized study. Int J Urol. 2015;22:967-71.

18. Aldaqadossi H, El Gamal S, El-Nadey M, El Gamal O, Radwan M, Gaber M. Dorsal onlay (Barbagli technique) versus dorsal inlay (Asopa technique) buccal mucosal graft urethroplasty for anterior urethral stricture: a prospective randomized study. Int J Urol. 2014;21:185-8.

19. Dubey D, Vijjan V, Kapoor R, Srivastava A, Mandhani A, Kumar A, et al. Dorsal onlay buccal mucosa versus penile skin flap urethroplasty for anterior urethral strictures: results from a randomized prospective trial. J Urol. 2007;178:2466-9.

20. Soliman MG, Abo Farha M, El Abd AS, Abdel Hameed H, El Gamal S. Dorsal onlay urethroplasty using buccal mucosa graft versus penile skin flap for management of long anterior urethral strictures: a prospective randomized study. Scand J Urol. 2014;48:466-73.

21. Alsikafi NF, Eisenberg M, McAninch JW. 317: Long-Term outcomes of Penile Skin Graft Versus Buccal Mucosal Graft for Substitution Urethroplasty of the Anterior Urethra. J Urol. 2005;173 (4S):87-8.

22. Raber M, Naspro R, Scapaticci E, Salonia A, Scattoni V, Mazzoccoli B, et al. Dorsal onlay graft urethroplasty using penile skin or buccal mucosa for repair of bulbar urethral stricture: results of a prospective single center study. Eur Urol. 2005;48:1013-7.

23. Wessells $\mathrm{H}$, McAninch JW. Current controversies in anterior urethral stricture repair: free-graft versus pedicled skin-flap reconstruction. World J Urol. 1998;16:175-80. 
24. Lumen N, Oosterlinck W, Hoebeke P. Urethral reconstruction using buccal mucosa or penile skin grafts: systematic review and meta-analysis. Urol Int. 2012;89:387-94.

25. Yeung LL, Brandes SB. Urethroplasty practice and surveillance patterns: a survey of reconstructive urologists. Urology. 2013;82:471-5.

26. Bertrand LA, Voelzke BB, Elliott SP, Myers JB, Breyer BN, Vanni AJ, et al. Measuring and Predicting Patient Dissatisfaction after Anterior Urethroplasty Using Patient Reported Outcomes Measures. J Urol. 2016;196:453-61.

27. Baradaran N, Fergus KB, Moses RA, Patel DP, Gaither TW, Voelzke BB, et al. Clinical significance of cystoscopic urethral stricture recurrence after anterior urethroplasty: a multiinstitution analysis from Trauma and Urologic Reconstructive Network of Surgeons (TURNS). World J Urol. 2019;37:2763-8.

28. Faris SF, Myers JB, Voelzke BB, Elliott SP, Breyer BN, Vanni AJ, et al. Assessment of the Male Urethral Reconstruction Learning Curve. Urology. 2016;89:137-42.

29. Fossati N, Barbagli G, Larcher A, Dell'Oglio P, Sansalone S, Lughezzani $G$, et al. The Surgical Learning Curve for One-stage Anterior Urethroplasty: A Prospective Single-surgeon Study. Eur Urol. 2016;69:686-90.
30. Benson CR, Goldfarb R, Kirk P, Qin Y, Borza T, Skolarus TA, et al. Population Analysis of Male Urethral Stricture Management and Urethroplasty Success in the United States. Urology. 2019;123:258-64.

31. Barbagli G, Palminteri E, Lazzeri M, Turini D. Interim outcomes of dorsal skin graft bulbar urethroplasty. J Urol. 2004;172(4 Pt 1):1365-7; discussion 1367.

32. Kessler TM, Schreiter F, Kralidis G, Heitz M, Olianas $R$, Fisch M. Long-term results of surgery for urethral stricture: a statistical analysis. J Urol. 2003;170:840-4.

\section{Correspondence address:} Steven B. Brandes, MD Department of Urology, Columbia Univeristy, 161 Ft. Washington Ave $11^{\text {th }}$ Floor New York, NY 10032, USA Telephone: +1 212 305-6151 E-mail: sbb2169@cumc.columbia.edu 
APPENDIX

Supplementary Figure 1 - Funnel Plots indicating the presence of publication bias among the studies for (a) all included studies in the analysis (b) studies using the failure definition of need for instrumentation (c) studies using the failure definition of recurrent stricture on routine urethrography or cystoscopy.

A

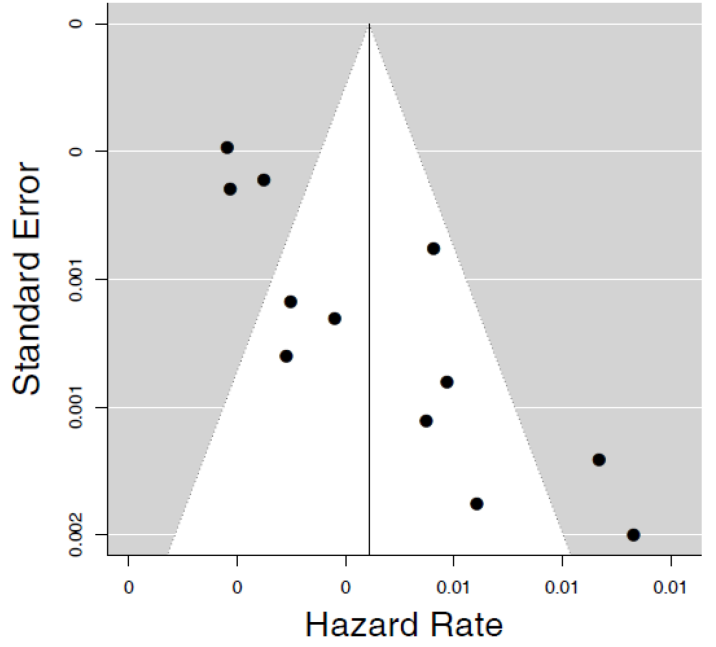

B

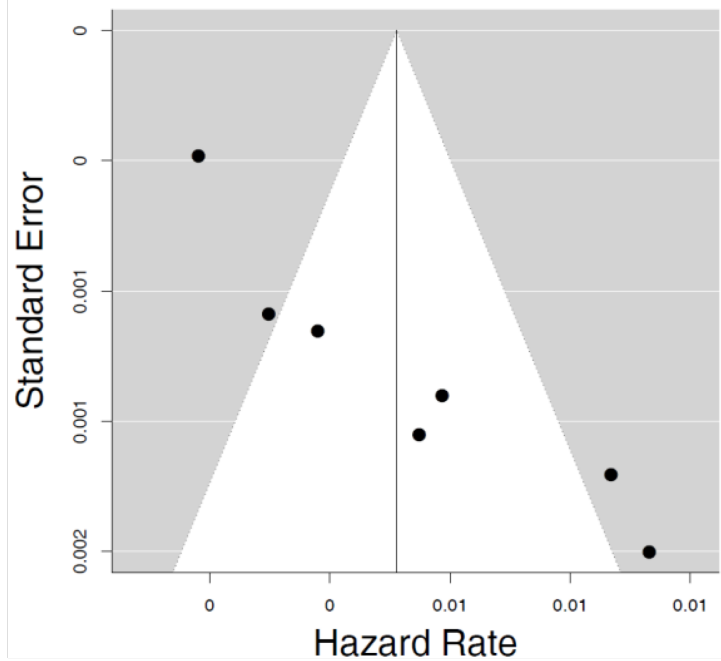

B

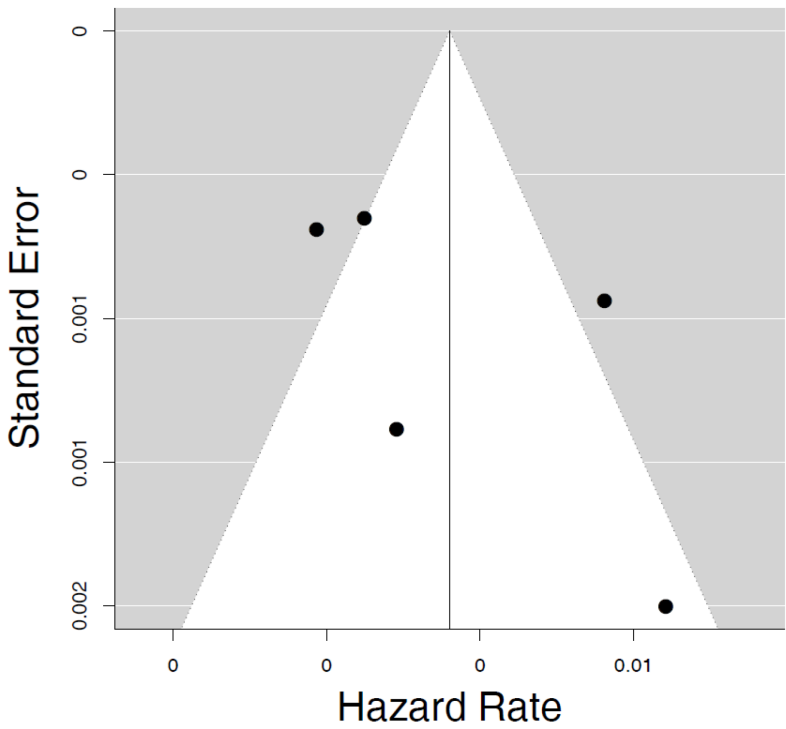


Supplementary Table 1 - Summary statistics and analysis of the studies included for meta-analysis. This includes the study population, age, definition of failure, follow-up, and etiology.

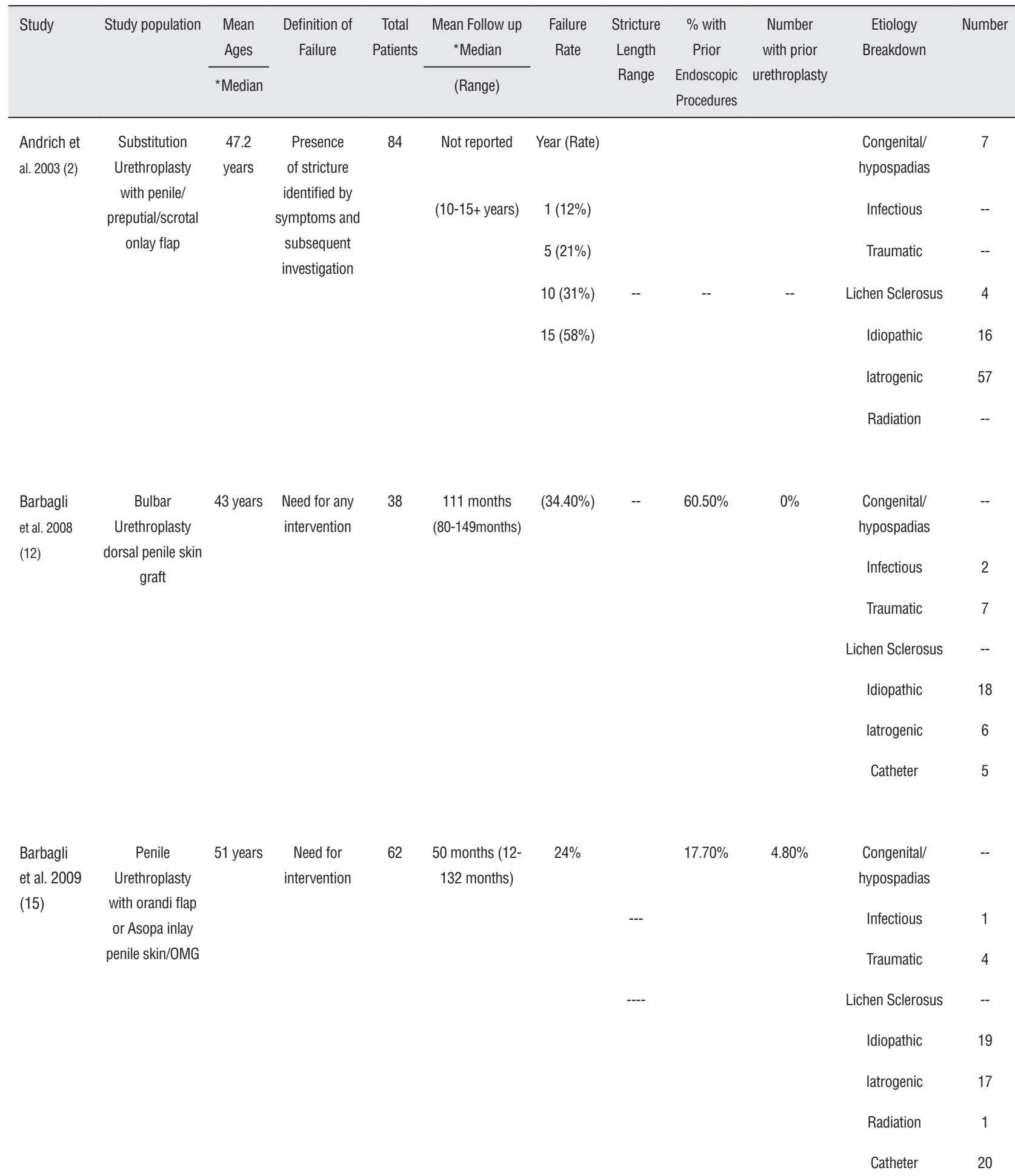




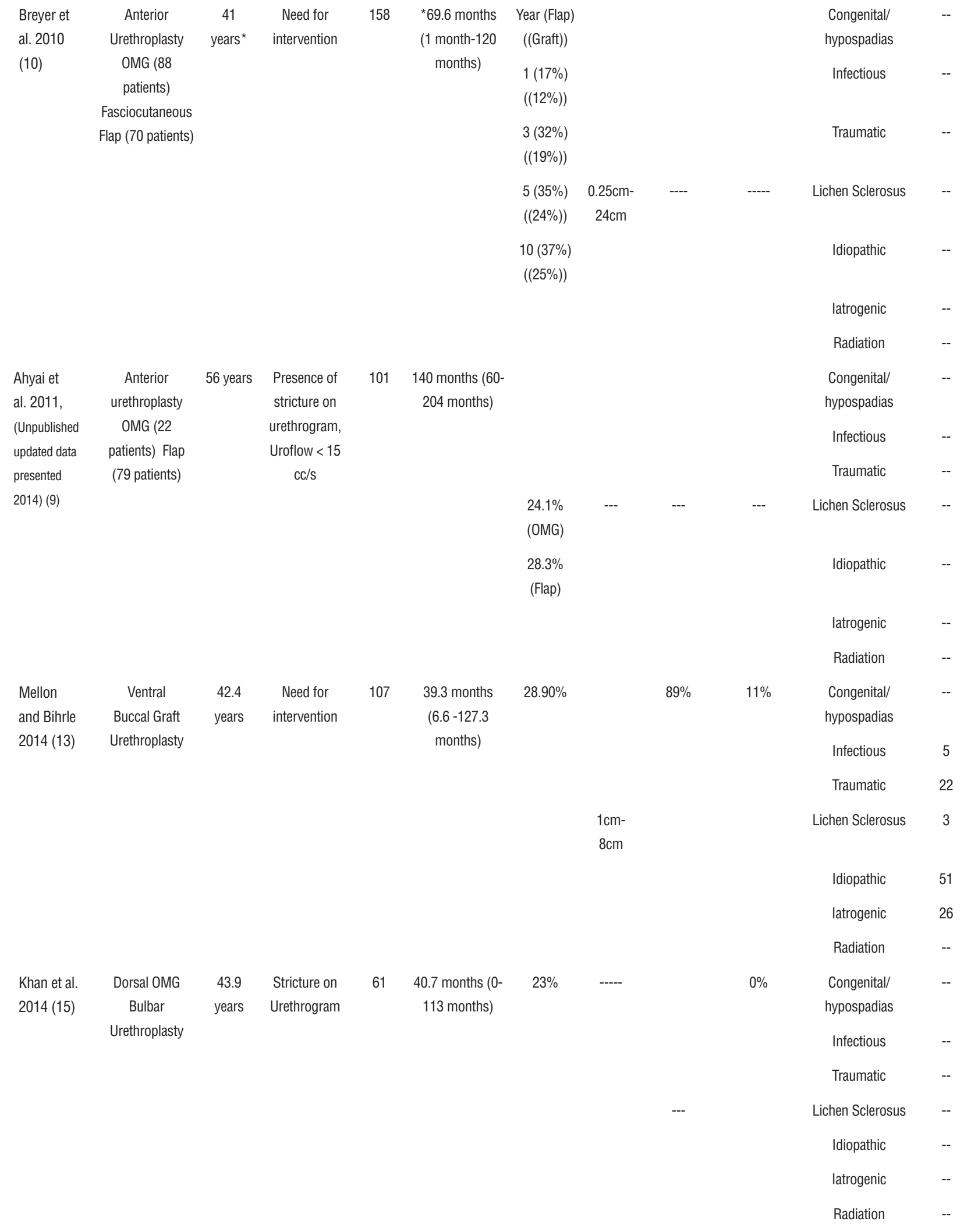




\begin{tabular}{|c|c|c|c|c|c|c|c|c|c|c|c|}
\hline $\begin{array}{l}\text { Palminteri } \\
\text { et al. } 2015\end{array}$ & $\begin{array}{c}\text { Bulbar } \\
\text { Urethroplasty }\end{array}$ & 40 years & $\begin{array}{l}\text { Need for } \\
\text { intervention }\end{array}$ & 166 & $\begin{array}{l}{ }^{*} 59.3 \text { months } \\
\text { (IQR 33-95.5 }\end{array}$ & $10.20 \%$ & & $75 \%$ & $1.20 \%$ & $\begin{array}{l}\text { Congenital/ } \\
\text { hypospadias }\end{array}$ & -- \\
\hline (6) & Overlappying & & & & months) & & & & & Infectious & -- \\
\hline & $\begin{array}{l}\text { Dorsal plus } \\
\text { ventral OMG }\end{array}$ & & & & & & & & & Traumatic & 7 \\
\hline & & & & & & & $1.9 \mathrm{~cm}-$ & & & Lichen Sclerosus & -- \\
\hline & & & & & & & $3 \mathrm{~cm}$ & & & & \\
\hline & & & & & & & & & & Idiopathic & 117 \\
\hline & & & & & & & & & & latrogenic & 8 \\
\hline & & & & & & & & & & Catheter & 34 \\
\hline Spencer & Anterior & 56 years & Need for & 73 & 44 months (12- & $12.30 \%$ & & $92 \%$ & $14 \%$ & Congenital/ & -- \\
\hline et al. 2018 & Urethroplasty & & intervention & & 162 months) & & & & & hypospadias & \\
\hline (11) & dorsal onlay & & & & & & & & & Infectious & -- \\
\hline & OMG, (Kulkarni) & & & & & & & & & & \\
\hline & for long segment & & & & & & & & & Traumatic & 8 \\
\hline & strictures & & & & & & $8 \mathrm{~cm}-$ & & & Lichen Sclerosus & 28 \\
\hline & & & & & & & $21 \mathrm{~cm}$ & & & & \\
\hline & & & & & & & & & & Idiopathic & 18 \\
\hline & & & & & & & & & & latrogenic & 16 \\
\hline & & & & & & & & & & Radiation & 3 \\
\hline Pandey et & Ventral & 51 & Presence & 104 & *54 months (1- & $9.60 \%$ & $1 \mathrm{~cm}-$ & & $100 \%$ & Congenital/ & -- \\
\hline al. 2017 & Onlay OMG & years* & of Stricture & & 205 months) & & $23 \mathrm{~cm}$ & & & hypospadias & \\
\hline (16) & Urethroplasty & & on Cysto/ & & & & & & & Infectious & $\ldots$ \\
\hline & & & Urethrogram & & & & & & & & \\
\hline & & & & & & & & & & Traumatic & -- \\
\hline & & & & & & & & _ & & Lichen Sclerosus & -- \\
\hline & & & & & & & & & & Idiopathic & -- \\
\hline & & & & & & & & & & latrogenic & -- \\
\hline & & & & & & & & & & Radiation & -- \\
\hline
\end{tabular}

